\title{
Glycaemic control at risk? - Impact of temperature, humidity and other physical factors on the analytical quality of point of care testing of blood glucose, a systematic review protocol
}

\section{Cilia Maria Katharina Sindt}

University College South Denmark: UC Syddanmark

Lene Noehr-Jensen ( $\square$ leno@ucl.dk)

University College Lillebaelt: UCL Erhvervsakademi og Professionshojskole https://orcid.org/00000001-5176-819X

\section{Research Article}

Keywords: Blood glucose meters, point of care testing (POCT), quality, physical settings, environmental conditions, glycaemic control

Posted Date: July 6th, 2021

DOI: https://doi.org/10.21203/rs.3.rs-678622/v1

License: (c) (1) This work is licensed under a Creative Commons Attribution 4.0 International License.

Read Full License 


\section{Abstract \\ Background}

Diabetes is a chronic metabolic disease characterized by increased blood glucose concentration. Selfmonitoring of blood glucose (SMBG) is a corner stone in lowering the risk of vascular complications of diabetes and requires reliable measurements by the blood glucose meter and test strips (BG System). Environmental factors as temperature, humidity, altitude, and electromagnetic radiation may affect the measurement of blood glucose to a level that might compromise the glycaemic control. Manufactures often restrict the validation and approved use a limited span of environmental conditions and there is a considerable risk of exposing the equipment to temperature outside the range recommended by manufacturers. This systematic review aims to explore, aggregate, and present the current knowledge of the effect of environmental factors on the quality of results measured by BG systems.

\section{Methods}

Relevant studies will be found by using a comprehensive search strategy in the following databases: EMBASE, PubMed, CINAHL, Academic Search Premier, SweMed+, DANS Easy and Cochrane Library. The search strategy will assess if there is an impact on precision and accuracy of BG systems under varying environmental conditions compared to precision recommended by manufacturers. Both authors will screen titles and in case of disagreement, the study will be included for screening by abstract. After screening of all titles, the procedure will be repeated for screening and reading of abstracts. Full text of papers selected by abstract will be assessed according to in- and exclusions criteria. Homogenous results will be pooled and summarized statistically for meta-analysis. Narrative synthesis will be performed for heterogeneous data that cannot be pooled for meta-analysis. Reporting will follow the Preferred Items for Systematic Reviews and Meta-Analysis 2020 (PRISMA 2020). The risk of bias will be evaluated by choosing an appropriate tool from acknowledged institutions I.e., Joanna Briggs Critical Appraisal for Systematic Reviews Checklist tools or the Critical Appraisal Skills Program (CASP).

\section{Discussion}

This systematic review protocol utilizes rigorous methodology to provide a comprehensive search for outcomes of the impact of environmental factors on BG systems. The outcomes will be examined, and a summary of the current knowledge will be provided.

\section{Background}

Diabetes is a chronic metabolic disease characterized by increased blood glucose (BG) concentration. The global prevalence of diabetes has increased dramatically over the last five decades and this development is predicted to continue in the future. (1) In 1964, it was estimated that 30 million people 
had diabetes. The International Diabetes Federation (IDF) estimates the number of people with diabetes in 2015 to 415 million and predicts the number to rise to 642 million by 2040 . $(1,2)$

Diabetes is highly associated with increased risk of morbidity and mortality and requires comprehensive efforts from the healthcare system. IDF estimated the total global health expenditure due to diabetes at 673 billion US dollars for 2015 and 802 billion US dollars for 2040. Furthermore, it was estimated that 5.0 million deaths in people aged 20-79 years were attributable to diabetes in 2015. (2)

Intensive glycaemic control lowers the risk of vascular complications of diabetes and post meal hyperglycaemia should be addressed by treatment with both non-pharmacologic and pharmacologic therapies $(2,3)$. Self-monitoring of blood glucose is a corner stone in this strategy and requires reliable measurements by BG systems consisting of BG meter and test strip.

A review by Erbach and colleagues published in 2016 revealed that environmental factors as temperature, humidity, altitude and electromagnetic radiation potentially affect the measurement of BG to a level that might compromise the glycaemic control. (4) Erbach et al emphasize that patients with diabetes should check the information on potential physical factors that might disturb the measurements of their BG systems, when planning a stay under extreme environmental conditions (4). However, manufactures often restrict the validation, approved use for a rather limited span of environmental conditions, and provide no information on the performance of the BG system under more extreme conditions. This leaves the patients with diabetes with few or no options to maintain the glycaemic control under these conditions. Further, extreme environmental conditions might occur unexpected and suddenly under certain circumstances. For example, transportation by car exposes the BG system to variable physical conditions. Studies have demonstrated that the temperature inside the cabin of a car exceeds the

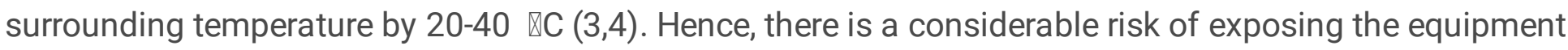
to temperature outside the range recommended by manufacturers.

Information of the impact of physical settings on the quality of measurements by BG systems are also highly relevant for both patients with diabetes and health care providers working with education and training of these patients in the large part of the world with more extreme climate conditions. People living in these areas might not have the opportunities of measuring BG under approved conditions, due to limited economic or technological resources.

Many modern BG systems have a built-in temperature sensor that allows a good performance under a broader range of temperatures, however rapid change in temperature still compromises the measurements from 15 to 30 minutes(4) and does not take the conditions for the test strips into account. Continuous glucose monitoring (CGM) systems are increasingly used.(2) They consist of a sensor placed on the skin of the patient, a data storage devise, and a monitor; the latter two parts are often integrated in the patient's mobile phone. CGM systems might potentially be a more reliable solution under extreme conditions, due to their protected position near the body. However, as these devices often require calibration with traditionally BG systems and most patients performing glycaemic control still use BG 
meters and strips this systematic review are focused to explore, aggregate, and present the present knowledge of the effect of physical settings on the quality of results measured by BG systems.

\section{Methods/design}

\section{Systematic review design}

Based on the clinical question: "Does physical factors e.g., temperature, humidity and altitude affect the analytical quality of Point of Care testing of blood glucose and does it affect the glycaemic control in individuals with diabetes?", a search strategy is developed for EMBASE and translated for each database to incorporate relevant participants, interventions, comparators, and outcomes (PICO) [see Additional file 1]. Studies published in English, Danish, Norwegian, Swedish and German are eligible for review.

Systematic review and subsequent analysis will be conducted following methods outlined in the PRISMA 2020 statement (5). A populated PRISMA-P checklist is provided [see Additional file 2] (6)

\section{Information sources and search strategy}

The following clinical electronic databases with noted limits on dates will be used to identify relevant literature using a systematic search strategy [see Additional file 1]:

EMBASE (2000 - present)

PubMed (2000 - present)

CINAHL (2000 -present)

Academic Search Premier (2000 - present)

SweMed+ (2000-2020)

DANS Easy -former OpenGrey (2000-present)

Cochrane Library (2000 - present)

The technological development of devices measuring blood glucose implies that it is not relevant to use literature published before 2000

To ensure literature saturation, the reference lists of included studies or relevant reviews identified through the search will be scanned for eligible studies.

\section{Population/participants included or excluded in search}

Studies on blood glucose test systems with no limit in country and both in-hospital and non-hospital settings are included. Studies conducted in laboratories simulating physical settings or studies using 
Quality control samples or artificial sample material will be included. Observational studies will also be included.

Participant using Continuous glucose monitoring systems (CSGMS) are not included in the study.

Animal studies are excluded.

\section{Interventions}

A broad range of blood glucose meters and matching test strips exposed to varying environmental conditions e.g., high/low temperature, humidity, or altitude. The interventions will be conditions that do not comply with the conditions approved by the manufacturers or conditions which are not within the testing scope of the manufacturer.

\section{Data management}

Zotero (7) will be used as the bibliographic management software. All found titles will be stored, duplicates will be removed, and all discarded references will be stored in a Zotero Archive.

\section{Selection process}

To achieve a high degree of consistency in the selection process both authors will discuss and adjust the common understanding of inclusion and exclusion criteria by screening the first 100 titles. Both authors will screen the following titles and in case of disagreement, the study will be included for screening by abstract. After screening of all titles, the procedure will be repeated for screening and reading of abstracts. The degree of agreement between authors will be quantified and reported in percentages. Full text of papers selected by abstract will be assessed according to in- and exclusions criteria.

\section{Data collection process and data items}

A specifically developed data extraction form will be used for studies found relevant to the selection criteria. Information will include as a minimum:

General details: Title, Authors, reference/source/year of publication, country, setting, study design, sources of funding/competing interest.

Eligibility criteria: POCT brand, analytical method, number of tests performed, intervention type (humidity, altitude, temperature)

Results: duration of testing period, sample size for each intervention type, Standard deviation, percent coefficient of variation (CV\%) and discrepancy

\section{Main Outcomes}

The following outcomes are evaluated: 
Estimation of precision under varying environmental condition compared to precision recommended by manufacturer or clinical guideline.

Estimation of accuracy under varying environmental conditions compared to accuracy recommended by manufacturer or clinical guideline.

The clinical impact of the estimated precision and accuracy will be assessed.

\section{Risk of bias}

Since the review question is focused on the analytical quality of glucose meters exposed to different physical factors, the selection of patient groups is not relevant. The risk of bias will be evaluated by choosing an appropriate tool from acknowledged institutions i.e. Joanna Briggs Critical Appraisal for Systematic Reviews Checklist tools or the Critical Appraisal Skills Programme (CASP).(8)

\section{Data analysis and synthesis of evidence}

Data will be presented in different types of ranked summary tables for each environmental condition evaluated. A performance summary table will be presented for each type of Glucometer included in the study. Homogenous results will be pooled and summarized statistically for meta-analysis. Narrative synthesis will be performed for heterogeneous data that cannot be pooled for meta-analysis. Reporting will follow the Preferred Items for Systematic Reviews and Meta-Analysis 2020 (PRISMA 2020). (5)

\section{Meta-Bias}

Histograms may be used to visualize and investigate the distribution of studies containing simulation of physical settings versus experiments in actual physical settings. Histograms may also be used to show the distribution of glucose meter characteristics.

\section{Confidence in cumulative evidence}

The quality of evidence for all outcomes will be judged using an adaption of the Grading of Recommendations Assessment, Development and Evaluation (GRADE) methodology (Grading of Recommendations Assessment, Development and Evaluation).

As a result of applying GRADE, the overall quality will be rated as:

High - further research is very unlikely to change our confidence in the estimate of clinical consequence.

Moderate - further research is likely to have an important impact on our confidence in the estimate of clinical consequence and may change the estimate.

Low - further research is very likely to have an important impact on our confidence in the estimate of clinical consequence and is likely to change the estimate. 
Very low - very uncertain about the estimate of clinical consequence.

\section{Discussion}

This systematic review protocol will utilize rigorous methods and pre-determined eligibility criteria to provide a uniquely comprehensive search for a broad spectrum of environmental factors impact on the quality of results measured on BG systems. The search strategy for this review was developed in consultation with content and methodological experts. We expect this review to cover an extensive part of the published knowledge of the subject as we use multiple databases and languages.

While our methodology is designed to minimize selection bias, we do anticipate limitations with this review due to methodological heterogeneity and the quality of existing studies. The broad spectrum of technologies and study designs will add to the amount of heterogeneity. Therefore, we anticipate that we may not be able to combine studies for a meta-analysis, but rather present findings using descriptive methods. Limitations of this review include the reliance on published data that may predispose to publication bias.

To our knowledge, this will be the broadest review of environmental factors on quality of blood glucose measurement. The review will provide insight into the clinical robustness of various available technologies and currently lacking data to guide device selection and utilization for patients.

\section{Abbreviations}

BG: blood glucose; CV\%: percent coefficient of variation; IDF: International Diabetes Federation

\section{Declarations}

\section{Ethics approval and consent to participate}

Not applicable

\section{Consent for publication}

Not applicable

\section{Availability of data and materials}

The datasets used and/or analysed during the current study are available from the corresponding author on reasonable request.

\section{Competing interests}

The authors declare that they have no competing interests 


\section{Funding}

The authors received no financial support for the research, authorship, and/or publication of this article

\section{Authors' contributions}

Both authors contributed equally to the conception, planning, analysis and writing of this article.

\section{Acknowledgements}

Helle Ernstsen Bach, Library and Information specialist, University College South Denmark.

Anne-Marie Fiala Carlsen, Library and Information specialist, UCL University College.

\section{References}

1. Entmacher PS, Marks HH. Diabetes in 1964: A World Survey. Diabetes. 1965 Apr 1;14(4):212-23.

2. Ogurtsova K, da Rocha Fernandes JD, Huang Y, Linnenkamp U, Guariguata L, Cho NH, et al. IDF Diabetes Atlas: Global estimates for the prevalence of diabetes for 2015 and 2040. Diabetes Res Clin Pract. 2017 Jun;1:128:40-50.

3. Leahy J (Jack) L, Aleppo G, Fonseca VA, Garg SK, Hirsch IB, McCall AL, et al. Optimizing Postprandial Glucose Management in Adults With Insulin-Requiring Diabetes: Report and Recommendations. J Endocr Soc. 2019 Oct 1;3(10):1942-57.

4. Erbach M, Freckmann G, Hinzmann R, Kulzer B, Ziegler R, Heinemann L, et al. Interferences and limitations in blood glucose self-testing: An overview of the current knowledge. J Diabetes Sci Technol. 2016;10(5):1161-8.

5. Page MJ, McKenzie JE, Bossuyt PM, Boutron I, Hoffmann TC, Mulrow CD, et al. The PRISMA 2020 statement: an updated guideline for reporting systematic reviews. BMJ. 2021 Mar 29;n71.

6. Moher D, Shamseer L, Clarke M, Ghersi D, Liberati A, Petticrew M, et al. Preferred reporting items for systematic review and meta-analysis protocols (PRISMA-P) 2015 statement. Syst Rev. 2015 Jan 1;4(1):1.

7. Zotero. | Your personal research assistant [Internet]. [cited 2021 May 19]. Available from: https://www.zotero.org/.

8. Roth S. Research Guides: Systematic Reviews \& Other Review Types: Tools for Critical Appraisal [Internet]. [cited 2021 May 16]. Available from:

https://guides.temple.edu/systematicreviews/criticalappraisal.

\section{Supplementary Files}

This is a list of supplementary files associated with this preprint. Click to download. 
- AdditionalFile1.docx

- Additionalfile2.docx 